\title{
Modélisation de l'élargissement Stark dans les lasers $\mathrm{X}$
}

\author{
N. Ben Nessib, Z. Ben Lakhdar, H. Nguyen* et J.P. Arranz* \\ Physique Atomique et Moléculaire, Faculté des Sciences de Tunis, 1060 Campus Universitaire, \\ Tunis, Tunisie \\ * Spectronomie des Gaz et des Plasmas, Université Pierre et Marie Curie, 4 place Jussieu, \\ 75252 Paris cedex 05, France
}

\begin{abstract}
Résumé : Nous présentons dans ce travail un code d'élargissement électronique où les collisions fortes sont traitées quantiquement et les collisions faibles par le formalisme semiclassique.
\end{abstract}

\section{INTRODUCTION}

Dans le cadre du développement des lasers $X$, des amplifications de rayonnement ont été observées expérimentalement par recombinaison d'ions lithiumoïdes au sein d'un plasma chaud. Des études expérimentales $(1,2,3)$ ont montré en particulier un gain pour les raies $5 f-3 d$ et $4 \mathrm{f}-3 \mathrm{~d}$ de l'Al XI.

Le pompage par recombinaison utilise des transitions ou le profil spectral peut être suffisamment modifié par l'élargissement Stark (4), l'étude des lasers X nécessite donc un développement des modèles théoriques de calcul de profil de raies dans les plasmas. Les modèles théoriques utilisent généralement l'approximation statique pour les ions et l'approximation d'impact pour les électrons $(5,6)$. C'est dans ce cadre que nous présentons une méthode de calcul d'élargissement Stark d'ions multichargés où l'élargissement électronique est traité quantiquement par la méthode Coulomb-Born (7) pour les collisions proches et par un traitement semi-classique des collisions lointaines.

\section{THEORIE}

Pour un moment angulaire de l'électron perturbateur 1 inférieur à $l_{1}$, l'approximation CBII est utilisée (sans échange et avec la condition d'unitarisation de la matrice de collision). Quand $l$ est entre $l_{1}$ et $l_{D}$, la fonction semiclassique de collision $a(z)$ est utilisée. La section efficace totale s'écrit alors :

où :

$$
\sigma^{\mathrm{T}}=\sigma^{\mathrm{Q}}+\sigma^{\mathrm{S} C \mathrm{C}}
$$

$$
\sigma^{\mathrm{Q}}=\frac{\pi}{\mathrm{k}^{2}\left[\mathrm{l}_{\mathrm{a}}\right]} \sum_{l, l^{\prime}, L}\left|\left\langle v, 1_{\mathrm{a}} ; \mathrm{k}, \mathrm{l} ; \mathrm{L}|\mathrm{T}| \mathrm{v}^{\prime}, \mathrm{l}_{\mathbf{a}}^{\prime} ; \mathrm{k}, \mathrm{l}^{\prime} ; \mathrm{L}\right\rangle\right|^{2}
$$




$$
\sigma^{\text {S.C }}=\frac{8 \pi}{3}\left|<v, l_{\mathrm{a}}\right| \mathbf{r}\left|\mathbf{v}^{\prime}, 1_{\mathrm{a}}>\right|^{2}\left[\mathrm{a}\left(\mathrm{z}^{1}\right)-\mathrm{a}\left(\mathrm{z}^{\mathrm{D}}\right)\right]
$$

$a(z)=z\left[K_{0}(z) K_{1}(z)\right], z^{1}=\frac{\rho_{1} \Delta E_{v v^{\prime}}}{k}, z^{D}=\frac{\rho_{D} \Delta E_{v x^{\prime}}}{k}, \rho_{1} k^{2}=l_{1}\left(l_{1}+1\right)$ et $\rho_{D}$ le rayon de Debye. L'intégration sur l'énergie de l'électron incident est réalisé par une quadrature de Gauss-Laguerre et la moyenne sur le micro-champ ionique est traitée par la méthode APEX : Adjustable Parameter Exponential Approximation (8).

\section{APPLICATION A L'AI XI}

Le tableau suivant donnent les sections efficaces que nous avons calculé pour la transition $4 \mathrm{f}-3 \mathrm{~d}$, utilisant le formalisme quantique, en fonction de l'énergie cinétique de l'électron perturbateur en Rydberg.

\begin{tabular}{|c|c|c|c|}
\hline $\mathbf{k}^{2}$ & $\sigma(3 \mathrm{~d})$ & $\sigma(4 \mathrm{f})$ & $\sigma(4 \mathrm{f}-3 \mathrm{~d})$ \\
\hline 1 & 3.62 & 9.30 & 4.54 \\
\hline 5 & 4.49 & 11.27 & 4.47 \\
\hline 15 & 4.89 & 14.32 & 4.28 \\
\hline 30 & 5.15 & 15.13 & 4.09 \\
\hline 50 & 5.30 & 14.45 & 3.93 \\
\hline 80 & 5.37 & 13.87 & 3.79 \\
\hline 150 & 5.13 & 12.16 & 3.62 \\
\hline 450 & 4.15 & 7.30 & 3.59 \\
\hline
\end{tabular}

\section{CONCLUSION}

Dans ce travail, nous avons présenté un modèle de l'effet Stark pour les ions émetteurs de laser $\mathrm{X}$ ayant une charge élevée immergés dans un plasma dense et chaud.

\section{Références :}

[1] P. Jeagle, G. Jamelot, A. Carillon, A. Klisnick, A. Sureau et H. Guenou, J. Opt. Soc. Am. B 4, 563 (1987)

[2] J. C. Moreno, H. R. Griem, S. Goldsmith et J. Knauer, Phys. Rey. A 39, 6033 (1989)

[3] J. C. Moreno, H. R. Griem, R. W. Lee, J. F. Seely, Phys. Rev. A 47, 374 (1993)

[4] J. C. Moreno, in Spectral Line Shapes, Volume 6, edited by L. Frommhold et J.

W. Keto, (AIP Conf. n²16 New York 1991)

[5] A. Calisti, F. Khalfaoui, S. Stamm, B. Talin et. R. W. Lee, Phys. Rev. A 42, 5433 (1990)

[6] S. Alexiou, Phys. Rev. A 49, 106 (1994)

[7] H. Nguyen, J. P. Arranz, N. Ben Nessib et Z. Ben Lakhdar, in Spectral Lime Shapes, Volume 7, edited by R. Stamm et B. Talin (Nova, New York, 1993)

[8] C. Iglesias, J. Lebowitz et O. Mac Gowan, Phys. Rev. A 28, 1667 (1983) 